Schizophrene Störungen 



\section{Schizophrene Störungen}

\section{Tiefere Einsichten durch bildgebende Verfahren}

Herausgeber: G. Gründer, Aachen 
Bibliographische Information der Deutschen Bibliothek

Die Deutsche Bibliothek verzeichnet diese Publikation in der Deutschen Nationalbibliografie; detaillierte bibliografische Daten sind im Internet über <http://dnb.ddb.de> abrufbar.

ISBN 3-8055-797|-3

Alle Rechte vorbehalten. Ohne schriftliche Genehmigung des Verlags dürfen diese Publikation oder Teile daraus nicht in andere Sprachen übersetzt oder in irgendeiner anderen Form mit mechanischen oder elektronischen Mitteln (einschließlich Fotokopie, Tonaufnahme und Mikrokopie) reproduziert oder auf einem Datenträger oder einem Computersystem gespeichert werden.

(c) Copyright 2006 by S. Karger GmbH, Postfach, D-79095 Freiburg, und S. Karger AG, Postfach, $\mathrm{CH}-4009$ Basel

Printed in Germany on acid-free paper by Konkordia GmbH · Das Medienunternehmen, Bühl ISBN 3-8055-797I-3 


\section{Inhalt}

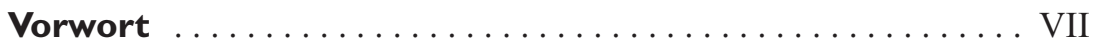

Gründer, G. (Aachen)

Interaktion von Genetik und Bildgebung $\ldots \ldots \ldots \ldots \ldots$. 1

Falkai, P.; Schneider-Axmann, T.; Wobrock, T.; Gruber, O. (Homburg/Saar)

Strukturelle Magnetresonanztomographie .......... 18 Meisenzahl, E.M. (München)

Magnetresonanzspektroskopie ................ 46

Braus, D.F.; Weber-Fahr, W. (Hamburg)

Funktionelle Bildgebung von Psychopathologie, Kognition und Emotionen $\ldots \ldots \ldots \ldots \ldots \ldots \ldots \ldots \ldots$

Kircher, T.; Habel, U. (Aachen)

Positronen-Emissions-Tomographie $\ldots \ldots \ldots \ldots \ldots \ldots 87$

Gründer, G. (Aachen)

Sachwortverzeichnis 



\section{Vorwort}

Mit der Entwicklung der Psychiatrie zu einer medizinischen Wissenschaft im 20. Jahrhundert war der Versuch, eine organische Basis für psychische Störungen zu identifizieren, nur ein folgerichtiger Schritt. Die enorme Komplexität des zu untersuchenden Organs und seine schlechte Zugänglichkeit machten dieses Unterfangen jedoch lange Zeit außerordentlich schwierig. Über Jahrzehnte war die Pneumenzephalographie die einzige Methode, einen - sehr groben Eindruck von der Struktur des lebenden menschlichen Gehirns zu gewinnen.

Durch die Entwicklung der modernen Schnittbildverfahren, Computertomographie (CT) und später Magnetresonanztomographie (MRT), wurde dieses invasive und in seiner Aussagekraft sehr eingeschränkte Verfahren überflüssig. Nun wurde es erstmals möglich, Details der Anatomie des lebenden menschlichen Gehirns zu erheben. Diese strukturellen bildgebenden Verfahren wurden in den letzten Jahren durch die funktionellen Verfahren ergänzt. Dazu zählen neben den nuklearmedizinischen Verfahren, Positronen-Emissions-Tomographie (PET) und Single-Photon-Emissions-Computer-Tomographie (SPECT), die funktionelle Magnetresonanztomographie (fMRT) sowie die Magnetresonanzspektroskopie (MRS). Erst diese ermöglichen die nicht-invasive Untersuchung biologischer Prozesse im lebenden Organismus. Mit der technischen Weiterentwicklung dieser Methoden, wie sie z.B. das Diffusion-Tensor-Imaging (DTI) oder die Real-Time-fMRT bieten, wird es möglich, immer subtilere Aspekte der Funktion des menschlichen Gehirns in vivo zu studieren.

Durch diese Methoden haben sich völlig neue Möglichkeiten der Einsicht in physiologische und krankhaft gestörte Hirnfunktionen eröffnet. Funktionelle bildgebende Verfahren haben im letzten Jahrzehnt neben molekularbiologischen Methoden den größten Erkenntnisgewinn in der Psychiatrie hervorgebracht. Während es vor wenigen Jahren z.B. noch üblich war, aus Konzentrationen von Neurohormonen auf die Funktion bestimmter, die Sekretion dieser Hormone steuernder Nervenzellen zu schließen, so erschließt sich diese heute in einer PET-Untersuchung unmittelbar. Damit wurde dem Neurowissenschaftler ein echtes «Fenster zum Hirn» geöffnet.

Schizophrene Störungen stellten von Beginn an eines der faszinierendsten Anwendungsgebiete der neuen Methoden dar, und auf kaum einem anderen Feld konnte ein vergleichbarer Zugewinn an Erkenntnissen erzielt werden. Heute ist es möglich, bestimmte Aspekte der Psychopathologie schizophrener Störungen definierten Hirnregionen zuzuordnen und deren Fehlfunktionen in 
vivo sichtbar zu machen. Die Verknüpfung mit modernen molekulargenetischen Methoden ermöglicht heute die Zuordnung individueller Unterschiede in bestimmten Hirnleistungen zu definierten genetischen Polymorphismen. Diese Entwicklung wird weitergehen. In absehbarer Zeit wird es möglich sein, die Funktion von Genen und die Expression ihrer Produkte am lebenden Gehirn zu studieren und sie zu gesunder und gestörter Hirnfunktion in Beziehung zu setzen.

Dabei sind die mit bildgebenden Verfahren erhobenen Befunde bereits heute nicht nur von akademischem Interesse. Einzelne Ergebnisse haben bereits Eingang in die tägliche klinische Praxis gefunden, oder sie beeinflussen in ganz erheblichem Maße die Entwicklung neuer Pharmaka. So machte Ende der 1980er Jahre die schwedische Arbeitsgruppe um Lars Farde mit der PET die Beobachtung, dass schon Dosierungen von 5-8 mg Haloperidol zu einer mehr als 80-prozentigen Blockade striataler Dopaminrezeptoren führen können, die mit einem erhöhten Risiko für extrapyramidalmotorische Nebenwirkungen einhergeht. Dies hat dazu geführt, dass heute erheblich niedrigere Dosierungen von Antipsychotika verordnet werden als noch vor 20 Jahren. Die pharmazeutische Industrie entwickelt heute kein Antipsychotikum mehr, ohne ihre neue Substanz vor dem breiteren Einsatz in klinischen Prüfungen an gesunden Probanden charakterisiert zu haben. Darüber hinaus werden mit Hilfe funktioneller bildgebender Verfahren Modelle für psychische Störungen entwickelt, an denen die Wirkung neuer Pharmaka überprüft werden kann. Tiermodelle für psychiatrische Erkrankungen werden damit nicht überflüssig, jedoch um eine wesentliche Erkenntnisquelle bereichert.

Dieses Buch soll all jenen, die mit Menschen arbeiten, die an schizophrenen Störungen leiden, nicht nur den aktuellen Wissensstand in diesem sich rasant entwickelnden Gebiet vermitteln; es soll sie auch in die Lage versetzen, sich durch ein Verständnis der angewandten Methoden die Primärliteratur erschließen zu können. Erst hierdurch entwickelt sich ein Sinn für die enormen Möglichkeiten, die sich den Neurowissenschaften in den nächsten Jahren erschließen werden. Bei aller damit verbundenen Euphorie darf jedoch nicht vergessen werden, dass bis heute ein enormer Graben klafft zwischen dem Wissen um die Physiologie und Pathophysiologie des menschlichen Gehirns und dem Verständnis, wie unser bewusstes Ich in diesem Organ zu lokalisieren ist. Es bleibt weiterhin ein kategorischer Unterschied zwischen unserem Wissen, welche Hirnregion aktiv ist, wenn unser Patient halluziniert, und dem Verständnis dafür, was diese Halluzinationen für seine individuelle bewusste Existenz bedeuten. So haben die modernen bildgebenden Verfahren eines ganz gewiss nicht bewirkt: die Entzauberung der Psyche als zutiefst humanistischem Konstrukt.

Aachen, im Dezember 2005 Gerhard Gründer 